\title{
La comprensión del valor de posición en el desempeño matemático de niños
}

\section{Understanding of Place Ualue in Children's Mathematic Performance f compreensão do valor de posição no desempenho matemático de crianças}

\author{
Diego Alonso Medina Rodríguez* \\ ${ }^{*}$ Universidad del Valle, Cali, Colombia.
}

Doi: http://dx.doi.org/10.12804/apl34.3.2016.01

\section{Resumen}

Este artículo presenta una revisión de los hallazgos teóricos más significativos obtenidos por estudios sobre la comprensión del valor de posición numérico en niños. La discusión realizada caracteriza y define el valor de posición, describe las habilidades y exigencias cognitivas relacionadas con la comprensión de este principio, e identifica los tipos de instrumentos más representativos para su evaluación, diagnóstico e intervención, así como el análisis de algunos modelos teóricos que explican el desarrollo de su comprensión. Los estudios revisados proponen que la comprensión del valor de posición implica la adquisición de estructuras conceptuales y procedimentales específicas, afectando tanto el desempeño matemático de los niños, como la escritura de numerales arábigos. Actualmente, la investigación del valor de posición intenta develar cómo ocurre el procesamiento numérico de este principio en numerales multidígitos.
Palabras clave: educación matemática; valor de posición; desarrollo; comprensión numérica; numerales.

\section{fibstract}

This article reports an overview of the most significant theoretical findings from research on the understanding of the place value of digits in children. It is proposed a discussion that characterizes and defines the object of study mentioned, describes the skills and cognitive demands related to their understanding, and identifies the most common types of tools used for assessment, diagnosis and intervention, as well as the analysis of some theoretical models that explain the development of understanding of the place value. The reviewed studies suggest that understanding of the place value implies the acquisition of specific conceptual and procedural structures, affecting children's performance in mathematics and writing Arabic numbers. Currently, the research

* Diego Alonso Medina Rodríguez, Grupo Matemática y Cognición, Centro de Investigaciones en Psicología, Cognición y Cultura, Universidad del Valle, Cali, Colombia.

La correspondencia relacionada con este artículo debe ser dirigida a Diego Alonso Medina Rodríguez, Centro de Investigaciones en Psicología, Cognición y Cultura, Universidad del Valle. Grupo Matemática y Cognición. Calle 13 No. 100-00, Edificio 385; Oficina 4007, 4to piso, Cali, Valle, Colombia. Correo electrónico: dialmero22@yahoo.com

Este documento es producto de la investigación: "Efecto de la comprensión del valor de posición en la escritura de numerales arábigos en niños de $1^{\circ}$ grado", avalado por la Universidad del Valle (C.I. 5209) y financiado por el Fondo de Becas Glen Nimnicht (Convenio 080 de 2009). Agradecimientos al Fondo de Becas Glen Nimnicht, por su apoyo para la realización de este documento.

Cómo citar este artículo: Medina Rodríguez, D. A. (2016). La comprensión del valor de posición en el desempeño matemático de niños. Avances en Psicología Latinoamericana, 34(3), 441-456. doi: http://dx.doi.org/10.12804/apl34.3.2016.01 
tries to unravel the place value as numerical processing of how this principle occurs in multi-digit numbers.

Keywords: mathematics education; place value concept; number concepts; number sense; numerals.

\section{Resumo}

Este artigo apresenta uma revisão dos resultados teóricos mais significativos obtidos por estudos sobre a compreensão do valor de posição numérico em crianças. A discussão realizada caracteriza e define o valor de posição, descreve as habilidades e exigências cognitivas relacionadas com a compreensão deste princípio e identifica os tipos de instrumentos mais representativos para a sua avaliação, diagnóstico e intervenção, assim como a análise de alguns modelos teóricos que explicam o desenvolvimento da compreensão do valor de posição. Os estudos revisados propõem que a compreensão do valor de posição implica a aquisição de estruturas conceituais e procedimentais específicas, afetando o desempenho matemático das crianças, assim como a escritura de numerais arábigos. Atualmente, a pesquisa do valor de posição tenta revelar como ocorre o processamento numérico deste princípio em numerais multidígitos.

Palavras-chave: educação matemática; valor de posição; desenvolvimento; compreensão numérica; numerais.

La relevancia del valor de posición como tema de estudio se fundamenta en el efecto que genera su comprensión sobre el desempeño matemático de los niños (Byrge, Smith, \& Mix, 2014). Chan y Ho (2010) plantean que el éxito en la resolución de sumas y restas se relaciona con la comprensión del valor de posición. Además, Hunter y Turner (1994) señalan que una inadecuada comprensión de este principio se traduce en dificultades para aplicar procedimientos de llevar y prestar en la resolución de problemas aritméticos, la comprensión y producción de numerales, etc., y que estas dificultades se incrementan progresivamente en cada nivel escolar.
Bedoya y Orozco (1991) sugieren que la caracterización del razonamiento infantil frente a la comprensión del valor de posición y del sistema de numeración en base diez (SNBD) facilita la generación de supuestos pedagógicos sobre el tipo de actividades que suscitan reflexión en los niños o la construcción de lineamientos conceptuales que sustentan un análisis adecuado de los desempeños observados. En este sentido, diferentes investigaciones han logrado identificar las nociones, operaciones y relaciones mentales que los niños ponen en funcionamiento al resolver tareas que involucran la aplicación de este principio (ver Baturo, 2000; Fuson, 1998; Price, 2001; Hiebert \& Wearne, 1992).

Este artículo pretende construir un panorama general sobre el estado de la discusión generada en torno al quehacer investigativo del valor de posición. Aunque los aportes en este campo de investigación son amplios, la discusión se orienta esencialmente sobre los hallazgos que permiten develar la demanda cognitiva que exige a los niños la comprensión de este principio y las dificultades que afrontan en su dominio. Para lograr este fin, se plantea: (a) definir y caracterizar el valor de posición; (b) examinar las principales habilidades y exigencias cognitivas relacionas con su comprensión; (c) identificar los instrumentos más representativos reportados en la literatura para la realización de procesos de evaluación, diagnóstico e intervención; (d) analizar algunos modelos que describen el desarrollo de la comprensión del valor de posición; (e) proporcionar algunas conclusiones frente al estado actual y futuro de su investigación.

\section{Caracterización del valor de posición}

El valor de posición es un principio organizador del SNBD que determina el valor numérico representado por un digito, el cual corresponde al valor nominal que lo identifica multiplicado por la potencia de diez asociada con la posición que ocupa en una cadena numérica (Price, 2001). Medina (2012) 
explica que cuando escribimos un numeral como el “462”, el dígito “4” en la tercera posición expresa cuatro unidades de cien que valen cuatrocientos, el dígito "6" señala seis unidades de diez que valen sesenta y el dígito " 2 " indica dos unidades de uno que valen dos. Orozco (1994) dice que el valor de posición no sólo representa el valor relativo al orden, sino también el valor relativo al número de unidades (que el dígito " 8 " en " 80 " equivale a 8 unidades de 10 u 80 unidades de 1). Así, Bedoya y Orozco (1991) proponen que la forma como se organiza el SNBD en unidades decimales sustenta la importancia de comprender el sistema jerárquico sucesivo e incluyente sobre el que opera para lograr una escritura de numerales exitosa. En este sentido, diferentes autores reportan cómo el valor de posición se encuentra intrínsecamente vinculado al SNBD y, por consiguiente, a las invariantes que lo caracterizan: composición/descomposición aditiva y multiplicativa, la equivalencia numérica, el concepto de unidad, etc. (ver Bedoya, 2013; Nunes \& Bryant; 1997; Saxton \& Cakir, 2006).

\section{La comprensión del valor de posición}

La comprensión numérica es caracterizada como un proceso continuo de carácter dinámico, conectivo y recursivo, que conlleva a la organización y reorganización de estructuras conceptuales (Meel, 2003). Lerner y Sadovsky (1994) proponen que la comprensión numérica plantea la apropiación de un objeto de conocimiento, el acceso a las reglas de funcionamiento que lo caracterizan y el establecimiento de vínculos entre representaciones. Ross (1990) relaciona la comprensión del valor de posición con la capacidad de coordinar y sintetizar conocimientos que tienen que ver con el SNBD. Ceballos (2012) y Price (2001) proponen que la comprensión del valor de posición involucra un carácter procedimental del conocimiento matemático y otro referido al contenido estructural de dicho conocimiento. Al respecto, Hiebert y Carpenter (1992) plantean que las estructuras conceptuales y procedimentales se generan o modifican constantemente a medida que los sujetos tienen acceso y manipulan diversos formatos de representación numérica. Price (2001) señala que la comprensión del valor de posición se evidencia cuando un sujeto es capaz de apropiarse de las características inherentes al valor de posición del SNBD, y utiliza de forma flexible y adecuada estructuras conceptuales para representar cantidades y escribir símbolos numéricos.

\section{Habilidades y exigencias cognitivas relacionadas con el valor de posición}

Nociones y habilidades diversas se vinculan con la comprensión del valor de posición. Por ejemplo, Boulton-Lewis (1993) explora la relación entre el conocimiento de la estructura de la secuencia de conteo y el valor de posición, aplicando una tarea a niños de $1^{\circ}, 2^{\circ}$ y $3^{\circ}$ grado; con ésta identificó que ellos generan secuencias de conteo más extensas de las aprendidas en el aula de clases y que las explicaciones que formulan a partir de las estructuras que identifican estas secuencias de conteo se relacionan con la comprensión que poseen del valor de posición. Esto se debe a que el dominio de la estructura que identifica la secuencia de conteo permite la comprensión de las relaciones parte-todo, la misma que es requerida para el conocimiento de la propiedad aditiva y que subyace a la representación del valor de posición en las cifras. Al respecto, Ross (1990) propone que las relaciones parte-todo son básicas en la comprensión de cifras de dos dígitos, pues este concepto se vincula con la inclusión de clases, es decir, la posibilidad de identificar que una colección —el todo — es mayor que cualquiera de las partes que la conforman.

Un estudio reciente, realizado por Chang, $\mathrm{Au}$ y Tang (2014) con niños de preescolar y $1^{\circ}$ grado, propone que el conteo es un predictor de la comprensión del valor de posición. Según esta investigación, las estrategias más sofisticadas de conteo se evidencian en los niños que presentan 
los mejores desempeños en la resolución de tareas matemáticas que involucran el uso de este principio. Otras investigaciones sugieren que los niños requieren de habilidades y nociones inherentes al SNBD como prerrequisito para una comprensión del valor de posición. Saxton y Cakir (2006) evalúan en niños de preescolar y $1^{\circ}$ grado la relación del "conteo a partir de", "la equivalencia numérica" y "la descomposición", con tareas que involucran la aplicación del valor de posición. Los resultados señalan que los niños no generan estrategias para la descomposición de cifras en términos de dieces y unos porque no establecen una comprensión del valor nominal y posicional de los dígitos; al parecer, estas dificultades se relacionan con la utilización de estrategias de conteo poco sofisticadas. La investigación también establece dificultades para diferenciar la noción de valor y cantidad, principalmente en las tareas que exigen establecer equivalencias numéricas. Finalmente, los autores concluyen que el "conteo a partir de", "la equivalencia numérica" y "la descomposición" son predictores del desempeño matemático de los niños en tareas relativas al SNBD, en especial "el conteo a partir de".

Aunque la relación entre el conteo y la comprensión del valor de posición parece sugerir beneficios para los procesos de aprendizaje de los niños, algunos estudios señalan límites para su utilización. Cotter (2000) encuentra que, al promover el aprendizaje del valor de posición a través del lenguaje y la visualización en niños de $1^{\circ}$ grado, el énfasis excesivo sobre el conteo puede llegar a generar interferencia en el desarrollo de las nociones vinculadas con el valor de posición. Por ejemplo, cuando un niño utiliza una estrategia que se fundamenta en el conteo de una colección de elementos unitarios, es probable que no pueda establecer una idea de conjunto, porque centrarse inicialmente de manera específica sobre la actividad de contar puede dificultar integrar las ideas sobre la naturaleza parte-todo de los numerales multidígitos, lo que puede generar que los niños crean que los numerales son sólo colecciones de unos.

Por otra parte, si bien es posible identificar investigaciones interesadas en abordar directamente el valor de posición y su relación con otras nociones inherentes al SNBD, la mayor parte de los hallazgos reportados derivan de estudios que evalúan la habilidad de los niños para escribir numerales arábigos. A continuación se discute y analiza este tipo de investigaciones.

\section{El valor de posición y la escritura de numerales}

La escritura de numerales arábigos exige a los niños en su aprendizaje el dominio de una serie de elementos conceptuales, códigos y reglas que se sustentan operatoriamente en la comprensión de la lógica del SNBD (Bedoya \& Orozco, 1991). Así, este aprendizaje no es fácil y los niños cometen errores al intentar traducir un número de un formato numérico a otro diferente, generando cifras con dificultades léxicas o sintácticas en la estructura que los caracteriza. No obstante, es precisamente el análisis de estas producciones erróneas lo que permite identificar dificultades en la comprensión del SNBD y del valor de posición, pues cada tipo de numeral genera exigencias cognitivas diferentes.

Orozco, Guerrero y Otálora (2007) describen los errores que niños de $1^{\circ}, 2^{\circ}$ y $3^{\circ}$ grado cometen al escribir numerales arábigos, reportando un tipo de error, entre otros, denominado yuxtaposición, que cosiste en la trascripción literal en numerales arábigos sucesivos de cada uno de los fragmentos que componen una expresión numérica verbal, por ejemplo, al dictar el numeral "trescientos cuarenta y cinco", los niños escriben "300405”. Este error revela una idea inicial sobre la composición aditiva del numeral arábigo que no se fundamenta sobre la comprensión del valor de posición, sino sobre el conocimiento que poseen sobre los numerales verbales y que genera la unión de elementos que 
resultan familiares. Medina (2013) propone que en $1^{\circ}$ grado los niños aplican simultáneamente una estrategia de carácter fonológico que consiste en la codificación literal de las expresiones numéricas verbales, lo cual conlleva a generar errores de yuxtaposición y de carácter posicional, que consiste en la producción de numerales sintácticamente correctos pero que evidencian dificultades léxicas. Así, la coexistencia de estas dos estrategias evidencian el conflicto cognitivo existente en cuanto al tipo de reglas que deben ser aplicadas en la producción de numerales arábigos.

Algunas investigaciones señalan que la comprensión que poseen los niños de los numerales depende de la estructura numérica que los identifica. Así, Sinclair y Scheuer (1993), al evaluar la comprensión que niños de preescolar y $1^{\circ}$ grado poseen sobre cifras de dos y tres dígitos, identifican que los niños utilizan estrategias como: (a) comparar la cantidad de dígitos de una cifra para establecer su magnitud; (b) establecer correspondencias entre la expresión numérica verbal y arábiga; (c) sumar los valores nominales de los dígitos para establecer el valor total de una cifra; (d) utilizar el valor nominal del dígito para establecer su valor posicional correcto.

Hederich y Camargo (2002) realizan un estudio con niños de $1^{\circ}$ a $3^{\circ}$ grado para observar el desarrollo de la escritura numérica, reportando que el valor de posición propone a los niños desafíos diferentes en cada rango numérico. Así, la escritura de cifras terminadas en cero es más fácil para los niños, porque su aprendizaje ocurre independientemente al rango de éstos. Además, plantean que los numerales de 3 y 4 cifras incrementan las dificultades sintácticas y que la escritura de este tipo de numerales implica comprender la función del cero intermedio. En este caso, los niños deben aprender que, aunque el " 0 " es un elemento que se expresa de manera implícita en el numeral verbal, tiene una forma gráfica efectiva en el numeral arábigo.

Várelas y Becker (1997) encuentran que niños de $2^{\circ}, 3^{\circ}$ y $4^{\circ}$ grado no identifican el valor de posición de los dígitos en una cifra y sólo reconocen su valor nominal, por ejemplo, establecen que el dígito "1" en 14 o 18 siempre vale " 1 ", pero no su valor total (que el "1" en 14 o 18 vale "10"). Los autores también sugieren que la comprensión del valor de posición depende de la adquisición conceptual de la función simbólica de lugar, más que de la comprensión procedimental generada en el intercambio de ítems numéricos (Becker \& Várelas, 1993).

La literatura igualmente reporta que los niños utilizan criterios diferentes para la escritura de cifras a medida que construyen una comprensión sobre el sistema de numeración arábigo y su lógica. Al respecto, Lerner y Sadovsky (1994) identifican los criterios aplicados por niños entre 5 y 8 años para la escritura de numerales: (a) juzgar a las cifras por el valor absoluto de sus dígitos, por ejemplo, los niños pueden llegar a considerar que " 46 " representa una cantidad mayor que " 123 " porque los valores nominales de los dígitos en el primer caso son mayores que en el segundo; (b) establecer que un numeral de tres dígitos puede ser mayor que uno de dos cifras, no por la cantidad que representa, sino porque posee un dígito de más; (c) determinar el valor de un numeral con la misma cantidad de dígitos en función del primer dígito que inicia la cadena numérica. Así, "234" resultaría ser mayor que " 123 ", porque el dígito inicial " 2 " del primer numeral es mayor que el dígito inicial " 1 " del segundo; (d) establecer la diferencia entre dos cifras con igual magnitud y que comparten los mismos dos dígitos iniciales, a partir de la comparación entre el valor nominal de los dígitos subsiguientes. Así, "2 345" es menor que "2 368", porque el " 4 " del primer numeral es menor que el " 6 " del segundo numeral. Igualmente, los autores identifican que los niños pueden operar sobre el valor absoluto de los dígitos, es decir, establecer por ejemplo que “789" es mayor que "1234", porque la suma del valor nominal de los dígitos en el primer caso es mayor que la suma de los dígitos en el segundo. 
Los estudios que exploran cómo las invariantes del SNBD afectan la comprensión del valor de posición también plantean hallazgos de interés. Nunes y Bryant (1997) indagan sobre si hay relación entre el desempeño que niños de $1^{\circ}$ y $2^{\circ}$ grado evidencian en tareas de composición aditiva y de escritura de numerales. Los resultados establecen grupos de niños que: (a) dominan la escritura de numerales y la composición aditiva; (b) dominan la composición aditiva pero no la escritura de numerales; (c) no dominan la composición aditiva ni la escritura de numerales. No sé encontraron niños que escriban numerales y no dominen la composición aditiva, por lo cual se propone que la composición aditiva es un predictor de una escritura correcta de numerales.

\section{Desarrollo y comprensión del valor de posición}

Se han propuesto diferentes aproximaciones a cómo ocurre el desarrollo de la comprensión del valor de posición. Así, algunos modelos explicativos postulan niveles, etapas o redes conceptuales, los cuales se sustentan en resultados obtenidos a través de la observación de grupos de niños con características diferentes o de cambios identificados en casos individuales. Price (2001) propone una caracterización de estos modelos a partir de cinco puntos específicos: (1) los niveles o etapas propuestos son caracterizados en función del grado de experticia logrado por los niños o la complejidad del tipo de comprensión observada; (2) se describen niveles o etapas de comprensión basadas en las conductas comunes que se identifican en los niños en un momento específico de tiempo; (3) los niveles o etapas se caracterizan por evidenciar secuencias de esquemas relacionadas con un estado inicial de inmadurez en la comprensión de los niños, el cual mejora progresivamente en cada nivel de representación superior; (4) los niveles o etapas se utilizan a menudo como un medio de comparación de los individuos y grupos de niños para describir las diferencias en el rendimiento y la comprensión;
(5) finalmente, aunque no existe un acuerdo sobre cómo ocurre el desarrollo de la comprensión del valor de posición, hay un acuerdo tácito sobre el tipo de estructuras internas que se identifican en los desempeños de los niños al resolver tareas que exigen aplicar el principio de valor de posición.

\section{El modelo triadico Ct-piv}

Fuson (1990a, 1998) plantea un modelo que describe una red de soporte conceptual necesaria para el desarrollo de la comprensión de los numerales multidígitos, la cual involucra estructuras conceptuales específicas concebidas como "categorías hipotéticas de actividad cuantitativa que parecen ser útiles en la comprensión de la enseñanza y el aprendizaje de un dominio" (Fuson, 1998, p. 50). Este modelo (denominado inicialmente modelo tríadico "UDSSI" y, según una revisión posterior, renombrado modelo tríadico "Ct-piv"; ver Fuson, 1998) establece diferentes estructuras conceptuales que usan los niños para significar las cifras. Así, cada concepción involucra el establecimiento de relaciones entre las palabras número, los numerales arábigos y las cantidades que corresponden a los formatos de representación de los números. Estas estructuras conceptuales no se desarrollan de manera simultánea y lineal sino progresivamente en la medida que los niños las generan al enfrentarse a diversas situaciones (Fuson et al., 1997). Igualmente, cuando aparecen concepciones nuevas, éstas no reemplazan las ya existentes, sino que simplemente se adicionan a las últimas.

La primera estructura conceptual es denominada "concepción de los multidígitos como elementos unitarios", derivada de la concepción de dígitos simples que los niños construyen a partir de su interacción con cantidades, palabras número y numerales escritos. Así, los elementos concretos no pueden ser diferenciados como unitarios cuando se encuentran dentro de agrupaciones. Además, las palabras número, al igual que los numerales arábigos, no son concebidos como integrados por 
partes sino como totalidades (e.g., las palabras numéricas "diez y seis" y los dígitos que conforman el numeral arábigo "16" no tienen referentes de cantidad separados).

El segundo tipo de estructura conceptual es denominado "concepción de dieces y unos", refiriéndose a la construcción inicial de la idea de los unos y dieces como clases diferentes. Fuson (1998) explica que la concepción de dieces y unos se construye a través de la identificación de regularidades en la utilización de las expresiones numéricas verbales y arábigas que estructuralmente son similares. Además, los niños advierten que en las secuencias numéricas de conteo que utilizan se generan ciclos repetitivos (e.g., "treinta y uno, treinta y dos... treinta y nueve, cuarenta, cuarenta y uno, cuarenta y dos... cuarenta y nueve, cincuenta"), y que en la escritura de las expresiones arábigas también se generan estas regularidades (e.g., 31, 32.. 39, 40, 41, 42... 49, 50). Así, los ciclos identificados en la secuencia de conteo y en la escritura de numerales, asociados además al uso de material concreto para conformar colecciones, permiten que los niños diferencien los unos y los dieces y relacionen las partes que conforman estos dos tipos de expresiones (e.g., que en la expresión numérica verbal "treinta y uno", el fragmento correspondiente a "treinta" se refiere a treinta objetos y el fragmento "tres" corresponde a tres objetos).

La "concepción de los unos y dieces como secuencias" es el tercer tipo de estructura conceptual. Este caso se relaciona con el reconocimiento de los dieces y unos como unidades que representan clases diferentes y que son simultáneamente incluyentes. Así, los niños pueden operar con la concepción de los dieces como un grupo conformado a partir de elementos unitarios, pero también como grupos diferentes de un mismo tipo, integrados por igual cantidad de elementos. Sin embargo, esto no implica que los niños identifiquen la cantidad de subgrupos que pueden llegar a conformar una colección (e.g., algunos niños no logran identificar en una colección de 50 elementos cuántos grupos de 10 podrían haber).

El cuarto tipo de estructura se denomina "concepción de los unos y dieces como elementos separados". En consecuencia, "en situaciones que proponen cantidades con grupos de diez, los niños comienzan a centrarse sobre el conteo de los grupos más que sobre los objetos que integran los grupos" (Fuson et al., 1997, p. 141). Así, los "unos" comienzan a designar colecciones que se transforman en elementos unitarios (e.g., "un" grupo de "diez", "dos" grupos de "diez") y los "dieces" dejan de ser concebidos como agrupaciones y se transforman en totalidades (e.g., "un diez"). Esto implica que los unos y dieces representan valores más que cantidades.

La "concepción de los dieces como la integración de secuencias separadas" corresponde al quinto tipo de estructura conceptual. Este caso se relaciona con el reconocimiento de los dieces como secuencias separadas de elementos unitarios (grupos diferentes conformados por diez elementos) y como elementos unitarios (un elemento con valor de diez), lo cual permite a los niños generar relaciones bidireccionales entre las palabras número, numerales arábigos y cantidades. El último tipo de estructura conceptual corresponde a la "concepción de los dígitos simples como elementos concatenados", el cual refiere a la idea del dígito como elemento unitario que toma un significado en función de la posición que ocupa en la cifra. Esta concepción se evidencia cuando los niños comienzan a trabajar con el algoritmo de la suma y de la resta.

Según Fuson (1990a), la comprensión del valor de posición involucra establecer las diferencias entre el sistema numérico verbal y el sistema numérico arábigo. A su vez, la comprensión de estos sistemas requiere la construcción de estructuras conceptuales multiunidad, que en sí corresponden a estados de conocimiento que se transforman a través de la progresiva reflexión de los niños 
sobre el conjunto de estructuras multidígitos. No obstante, la comprensión del valor de posición requiere lapsos de tiempo extensos y la participación de los niños en actividades de carácter intensivo, que se apoyen en el uso de bloques de base diez, en la resolución de sumas y restas, para facilitar la construcción de estas estructuras conceptuales. Actividades de este tipo permiten que los niños sean capaces de corregir por sí mismos sus errores de escritura (Fuson \& Briars, 1990) y facilitan clarificar e identificar características del valor de posición, tales como: (a) las regularidades en la composición y descomposición de las unidades del sistema en grupos y subgrupos de diez; (b) la necesidad de significar el cero y su uso correcto en cada posición del numeral; (c) identificar los cambios en los valores de los dígitos en función de su posición; (d) generar números de gran magnitud mediante el intercambio y adición continua de dígitos hacia la izquierda del numeral (Fuson, 1990b).

\section{Modelo sobre estructuras cognitivas conexas}

Baturo (2000) propone un modelo que consta de tres "niveles" de conocimientos jerarquizados e incluyentes, que son requeridos para la comprensión del valor de posición en el SNBD. Aunque este modelo es utilizado para describir el proceso de comprensión de los números decimales, también permite establecer la relación entre las estructuras cognitivas necesarias para la comprensión de los números naturales.

El primer nivel de conocimiento se encuentra relacionado con la posición, la base del sistema numérico y el orden, estructuras que fundamentan la linea de conocimiento de base necesaria para la comprensión de los números decimales. La posición se encuentra asociada al conocimiento semántico necesario para identificar el nombre de un dígito en función de una posición particular (e.g., "2,53" $\rightarrow$ cinco décimas, tres centésimas, dos unidades) y del respectivo orden de posición de cada uno de los dígitos que integran el ítem.

El orden se refiere a la organización sintáctica específica de los dígitos en un numeral. Esta organización no es dependiente de la dirección "izquierda-derecha" utilizada en la escritura de números naturales, porque en los numerales decimales los valores que expresan los dígitos que se encuentran más hacia la derecha corresponden a valores cada vez más reducidos. Así, en los numerales decimales la noción de orden permite el reconocimiento tanto de la posición que ocupan los dígitos en una cifra como de cuándo estos pueden ser intercambiables (e.g., "2,53" $\rightarrow$ cinco décimas, tres centésimas, dos unidades). Además, el significado que estos adquieren en la cadena numérica no se modifica cuando se agrega un cero a la izquierda o a la derecha del numeral (e.g., el significado de $2,53 \rightarrow$ 02,53 y 2,530).

La base corresponde a la forma de organización del sistema de unidades en términos de cómo se agrupan, dividen y relacionan entre sí, para constituir súperunidades y subunidades con magnitudes diferentes. En el SNBD las unidades pertenecientes a una misma clase - independientemente del valor que expresen - se agrupan y son divisibles en diez elementos. Además, en los numerales decimales la noción de base se encuentra asociada con la orientación izquierda-derecha. Así, los dígitos más hacia la derecha expresan unidades diez veces menores que aquellos que se encuentran más hacia la izquierda, y los dígitos que se encuentran más hacia la derecha expresan unidades diez veces mayores que aquellos ubicados más hacia la izquierda.

El segundo nivel en el modelo involucra la unitización y la equivalencia, procesos necesarios para la comprensión de los números decimales y que corresponden al conocimiento vinculante requerido para desarrollar estructuras del tercer nivel. Lamon (1999) explica que la unitización es el "proceso" de construir unidades de referencia, lo que se relaciona con el reconocimiento de un tipo 
de unidad específico en una situación o problema particular - identificación de unidades simplespara reinterpretar dicha situación a partir del tipo de unidad establecido. Así, cuando una unidad compuesta se forma (e.g., 1 caja de colores), un problema puede ser reinterpretado en términos de dicha unidad, parte/todo (e.g., 32 colores componen 1 caja de colores). Sin embargo, un problema puede exigir reconceptualizar la unidad establecida en función de las relaciones que establece con otros tipos de unidades -identificación de unidades compuestas - ; en estos casos se habla de una reunitización (e.g., 1 paquete de colores puede descomponerse en 10 cajas de colores, que a su vez pueden descomponerse en 32 colores) y de equivalencia, la cual hace referencia al proceso de comparar y establecer relaciones de igualdad entre dos números. El tercer nivel comprende la estructura aditiva, estructura multiplicativa y la reunitización, que permiten generar la integración de todos los niveles de conocimiento. Este nivel de conocimiento es denominado "conocimiento estructural".

Finalmente, el modelo propone que para la construcción de un numeral, un niño debe tener la habilidad de comprender las cifras como una totalidad y reconocer la relación entre cada una de sus partes. Esta habilidad se fundamenta en la noción de unidad, la cual se construye a partir de la composición, descomposición y recomposición de unidades simples y compuestas — números pequeños y grandes - y de la identificación de las relaciones que se generan entre éstas, las cuales se operacionalizan a partir del dominio de las estructuras aditivas y multiplicativas.

Por otra parte, modelos adicionales a los mencionados aparecen reportados en la literatura, por ejemplo, la red de apoyo instruccional para el fortalecimiento de la comprensión de los numerales multidígitos y del valor de posición (Jones, Thornton, \& Putt, 1994). Este modelo explica cómo ocurre el desarrollo de la conceptualización de los numerales multidígitos y del valor de posición a partir de la identificación de cuatro habilidades básicas que evolucionan paralelamente con la comprensión del sistema decimal: contar, desagrupar, agrupar y relacionar números. El modelo de desarrollo de la comprensión del valor de posición (Ross, 1986) describe en cinco etapas cómo ocurre el proceso de adquisición de las estructuras de conocimientos necesarias para la comprensión de los numerales multidígitos. Además, está el modelo de representaciones mentales de los numerales multidígitos y la comprensión del valor de posición (Miura, Okamoto, Kim, Steere, \& Fayol, 1993). No obstante, aunque estos modelos realizan aportes interesantes, su discusión excede las posibilidades de presentarlos a profundidad en este documento y, por ello, sólo se incluyeron los más representativos.

\section{Evaluación, diagnóstico e intervención en la comprensión del valor de posición}

Las investigaciones en comprensión del valor de posición plantean la aplicación de diferentes tipos de instrumentos, los cuales aluden a modelos de representación físicos, numéricos o virtuales. Estos instrumentos se aplican para realizar procesos de evaluación, diagnóstico o intervención, dependiendo del objetivo trazado por el estudio. Así, un primer tipo corresponde a tareas que proponen la utilización de material concreto (ver Bedoya, Muñoz, \& Medina, 2012; Broadbent, 2004). Un segundo tipo involucra la escritura de numerales (ver Chang et al., 2014; Orozco et al., 2007) o de algoritmos vinculados con las operaciones aritméticas (ver Cawley, Parmar, Lucas-Fusco, Kilian, \& Foley, 2007). Un tercer tipo corresponde a tareas que involucran el uso de Tecnologías de la Información y Educación (ver Cadavid, 2013; Price, 1999). No obstante, la aplicación de este tipo de tareas no son de carácter excluyente, ya que algunos estudios utilizan dos o más de los tipos mencionados. Así mismo, las tareas utilizadas pueden corresponder a situaciones de resolución de problemas en contextos significativos o carecer de estas características. 
Kamii (1985) aplica una tarea de correspondencia de dígitos a niños entre 4 y 9 años para evaluar el nivel de comprensión que logran del valor de posición. Esta tarea consiste en proporcionar una colección con 16 fichas que deben ser contadas y dibujadas individualmente en una hoja de papel. A continuación, se solicita la escritura del numeral "16" y luego se pregunta sobre el significado de cada dígito (generalmente empezando por el dígito que representa las unidades), mientras se encierra con un círculo el dígito señalado. Finalmente, se encierra de manera conjunta en un nuevo círculo las partes que componen la cifra y se interroga al niño sobre las relaciones que identifica entre el numeral "16" y los dígitos "1" y "6". Los resultados establecen que los niños piensan que los dígitos en una cifra representan elementos unitarios. Así,

(1) el 6 de 16 significa 6 objetos, y el 1 del 16 significa un objeto. El hecho de que sobren 9 objetos no tiene ninguna importancia para estos niños. (2) El 6 de 16 significa un conjunto de seis, y el 1 del 16 un conjunto de un objeto. Los niños no establecen en ninguno de los dos casos una relación entre las partes numéricas (seis y diez objetos) y el todo numérico (dieciséis objetos) que es representado (Kamii, 1985, p. 65).

La autora concluye que la comprensión del valor de posición es muy compleja para los niños y que ellos deben primero construir nociones como la unidad simple y compuesta, antes de ser introducidos en el aprendizaje de este principio.

Las tareas de correspondencias de dígitos, aplicadas generalmente de manera individual, también han sido adaptadas a situaciones de resolución de problemas. Ross (2002) realiza un estudio con el fin de explorar el efecto por instrucción generado en la comprensión que niños de $3^{\circ}, 4^{\circ}$ y $5^{\circ}$ grado evidencian sobre numerales de dos y tres cifras, cuando se desempeñan en tareas de correspondencia de dígitos. Esta tarea consiste en facilitar a cada estudiante una hoja de papel con un gráfico de 35 frijoles organizados en un rectángulo que describe una matriz de $5 \times 7$. Una vez que los niños de manera grupal establecen la cantidad total de frijoles presentes en el dibujo, el investigador escribe el numeral " 35 " e insta a cada niño a realizar la misma acción sobre una transparencia que recubre la hoja de papel proporcionada, mientras pregunta sobre la relación del dígito " 5 " con la colección de frijoles. Después realiza el mismo procedimiento con el dígito " 3 ", por ejemplo, "esta parte del treinta y cinco, ¿con cuántos frijoles en el dibujo tiene que ver? Escribe lo que piensas y colorea los frijoles para explicar lo que quieres decir" (Ross, 2002, p. 421). Los resultados muestran que, tras el proceso de intervención, el éxito en las respuestas de los niños se incrementa en un $70 \%$, lo que sugiere que los niños mejoran en su comprensión del significado de los dígitos en los numerales.

Por otra parte, Várelas y Becker (1997) proponen que las tareas de intervención fundamentadas en manipulativos que expresan propiedades del SNBD no son suficientes para que los niños comprendan el valor de posición, al no permitir modelar la idea de que un mismo símbolo numérico como "4" puede llegar a ser utilizado para representar valores diferentes. Estos autores desarrollaron una tarea para trabajar con niños de $3^{\circ}, 4^{\circ}$ y $5^{\circ}$ grado que propone un sistema de signos con características semióticas específicas intermedias entre el valor de posición del SNBD y los manipulativos, el cual facilita operar dos aspectos fundamentales: (1) la multiplicidad del "valor" que se expresa en el valor nominal de los dígitos; (2) la composición de los valores que permite identificar el valor total de los dígitos en un numeral. La tarea consta de una serie de fichas y un tablero dividido en columnas que representan las unidades del sistema. Así mismo, cada ficha posee un color diferente en sus dos caras $y$, a su vez, cada cara expresa un valor distinto que puede corresponder al valor nominal o valor total. Por ejemplo, si una ficha expresa en su cara superior el valor nominal correspondiente a " 3 ", en su parte inferior - que se encuentra oculta — puede 
expresar el valor total de " 3 " o "30" o " 300 ". La finalidad de la tarea es que los niños seleccionen las fichas correctas para representar cantidades y las ubiquen sobre el tablero dividido en columnas. En este tipo de tareas los niños se ven libres del valor posicional para realizar la composición de un numeral arábigo y éste sólo constituye un indicador secundario para identificar el valor total de la cifra. Los resultados del estudio indican que la instrucción y la práctica a partir de esta actividad facilitan a los niños establecer diferencias entre el valor nominal y el valor total de los dígitos en las representaciones numéricas multidígitos.

Así mismo, Price (1999) investiga el impacto que tiene la utilización de modelos físicos y virtuales en la conceptualización de objetos matemáticos en niños de 7 y 8 años en $3^{\circ}$ grado. El objetivo de este estudio es caracterizar las concepciones que los niños poseen sobre la comprensión del valor de posición en numerales de 2 y 3 dígitos. En total cinco tipos de tareas son utilizadas: (1) representación de números; (2) reagrupación-comparación; (3) ordenamiento de números; (4) conteo a partir de, y (5) operaciones de suma y resta. Estas tareas involucran el uso de bloques en base diez en dos presentaciones: adaptados a un programa de computador y material concreto, que los niños deben utilizar para representar numerales. Así, en un tablero se presenta un numeral que aparece dividido dígito a dígito en función de una serie de columnas que indican en orden convencional cada una de las tres primeras unidades del SNBD. Los niños deben componer y representar con los bloques en las columnas el valor que representa cada uno de estos dígitos y, además, realizar procedimientos de conteo de unos y dieces, comparar numerales arábigos, establecer agrupaciones de dieces, y sumar y restar para lograr la composición total del numeral. Los resultados en este estudio no señalan diferencias entre los dos formatos de representación utilizados, indicando que los modelos físicos y virtuales son efectivos en el desarrollo de la comprensión del valor de posición. Sin embargo, un análisis cualitativo de los procedimientos de conteo de los niños en ambos casos permitió establecer ventajas y desventajas en su utilización. Según Price (1999), el trabajo con programas de computador genera un mayor impacto en la conceptualización matemática de los niños al incrementar la eficiencia en los procedimientos que utilizan y disminuye la demanda cognitiva de las tareas propuestas, ya que se reduce el tiempo que los niños utilizan en el conteo de objetos y las exigencias de memorización. Al parecer, el programa libera a los niños del conteo y les permite concentrarse en otros aspectos matemáticos, más que en las representaciones de los bloques.

Por otra parte, algunas investigaciones plantean que cambios significativos en la comprensión del valor de posición pueden generarse en periodos cortos de tiempo, siempre y cuando las tareas faciliten a los niños reflexionar sobre la estructura de los numerales. Medina (2012), en un estudio con niños de $1^{\circ}$ grado, evalúa el efecto que la comprensión del valor de posición genera sobre la escritura de numerales arábigos de 2 y 3 dígitos. Este estudio involucra un proceso de intervención que plantea la aplicación de una tarea de composición aditiva y otra de equivalencia numérica, las cuales proponen a los niños reflexionar sobre la lógica operatoria del SNBD.

En la primera tarea se presentan unas fichas (tarjetas que representan las unidades del sistema: $1,10,100$, etcétera), que corresponden a un número específico y que deben ser introducidas según su valor en contenedores que representan diferentes órdenes numéricos. Así, en al primer contenedor sólo pueden introducirse fichas que valen 1 , en el segundo contenedor fichas que valen $10 \mathrm{y}$ en el tercero fichas que valen 100. En cada contenedor sólo puede introducirse una cantidad de nueve fichas de un mismo valor. En este sentido, el tamaño del contenedor está diseñado para que se genere esta restricción. Por ejemplo, si a un niño se le proporcionan en desorden tres fichas que valen 1 , seis fichas que valen $10 \mathrm{y}$ dos fichas que valen 100 , 
este debe organizar las fichas por su valor en cada contenedor, y establecer el valor de cada colección formada y el valor total de todas las colecciones. Una vez el niño ha realizado las diferentes composiciones aditivas, se le pide seleccionar de una serie de tarjetas que representan los operadores del SNBD (dígitos 1, 2, 3, 4, 5, 6, 7, 8, 9 y 0) aquellas que corresponden al valor de las colecciones que hay en los contenedores (e.g., si en el primer contenedor hay tres fichas, debería seleccionarse una tarjeta con el dígito " 3 ”); despues, se les pide ubicar cada tarjeta en un tablero con tres posiciones (que se encuentran demarcadas con tres líneas horizontales que representan los órdenes numéricos del sistema), estableciendo concordancias entre el valor total del dígito representado por cada tarjeta y el valor de cada colección, así como entre el valor nominal de los dígitos y la cantidad de fichas en cada contenedor.

Para la tarea de equivalencia numérica, presentada sólo a los niños que resuelven la tarea de composición aditiva, se incrementa la cantidad de fichas dadas (e.g., doce fichas que valen $1 \mathrm{y}$ diez que valen 10), de tal manera que deben realizar intercambios entre fichas de diferente valor (e.g., una ficha que vale 10 por diez que valen 1) para lograr cumplir la consigna de la tarea, que consiste en introducir en los contenedores la totalidad de las fichas proporcionadas.

Finalmente, los hallazgos encontrados en la investigación sugieren que la composición y la equivalencia son predictores de una escritura numérica exitosa y que cada tarea afecta de forma diferente la comprensión de los tipos de estructura que identifican un numeral arábigo. Además, los datos apuntan a que el proceso de intervención en composición genera efectos especialmente sobre el desempeño de los niños en tareas de comprensión y el proceso de intervención en equivalencia sobre el desempeño de los niños en las tareas de escritura de numerales.

\section{Conclusiones}

El valor de posición es caracterizado en la literatura como el principio organizador del SNBD. Diferentes investigaciones plantean que su comprensión afecta el desempeño matemático de los niños, incluyendo la escritura de numerales arábigos, por lo que su estudio es fundamental.

En la literatura se reporta cómo este principio se encuentra relacionado con habilidades y nociones numéricas como el conteo, la construcción de los números naturales, la comprensión de unidades simples y compuestas, el establecimiento de relaciones de equivalencia entre unidades, la composición y descomposición aditiva, etc. De la misma manera, algunos estudios plantean que la comprensión de algunas invariantes del SNBD, como el concepto de unidad, la composición aditiva, la equivalencia numérica, etc., son predictores de la comprensión del valor de posición.

Así mismo, es posible identificar cómo muchos estudios plantean un estrecho vínculo entre la comprensión del valor de posición y el dominio del algoritmo de las operaciones aditivas y multiplicativas, lo cual se debe a que tales operaciones constituyen la base sobre la que tradicionalmente se basa la enseñanza de este principio (ver Hiebert \& Wearne, 1996; Ho \& Cheng, 1997; Thompson, 2000). Al respecto, parece evidenciarse un supuesto que establece que el valor de posición debe enseñarse junto con el algoritmo de las operaciones aritméticas y no de forma simultánea con los procesos de escritura inicial, lo cual se fundamenta en el argumento de que al hacerlo se interfiere con la habilidad para resolver problemas aritméticos (Hugues, 1986). Sin embargo, Johansson (2005) establece que la adquisición de la secuencia de palabras numéricas y las habilidades para la escritura de numerales arábigos se correlaciona con un alto rendimiento en la resolución de problemas aritméticos, porque tales habilidades son la base para el 
desarrollo de una secuencia numérica mental que facilita la recuperación de procedimientos de solución. No obstante, la hipótesis mencionada continúa siendo revisada en el ámbito experimental.

Por otra parte, el desarrollo de la comprensión del valor de posición está relacionado con la adquisición de estructuras conceptuales y procedimentales específicas. Algunos modelos teóricos proporcionan una explicación de cómo surgen y se relacionan entre sí tales estructuras conceptuales, facilitando generar propuestas pedagógicas sobre el tipo de actividades que suscitan reflexión en los niños, y el planteamiento de lineamientos conceptuales que fundamenten el análisis de los desempeños observados.

Diferentes tareas y situaciones se proponen para promover una mejor comprensión del valor de posición y de los numerales multidígitos, pero se destacan principalmente las tareas de correspondencia de dígitos con material concreto vinculadas a situaciones de resolución de problemas, que constituyen una herramienta de intervención tradicional. El énfasis en el uso de tareas de este tipo se debe a que posibilitan evaluar la comprensión de los aspectos convencionales del valor de posición, y el valor total y nominal de los dígitos en una cifra. Al respecto, Cawley et al. (2007) proponen que el papel del valor de posición debe trascender el conocimiento posicional y que el diseño de tareas debería estar articulado a evaluar cómo promover una comprensión numérica. Además, en las investigaciones se evidencia una tendencia a incorporar tareas que involucran el uso de programas de computador como alternativa para observar y analizar los desempeños que generan los niños. Este tipo de herramientas ha permitido a los investigadores ampliar la perspectiva de observación de los fenómenos de aprendizaje, comprensión y desarrollo vinculados al estudio del valor de posición.

Finalmente, la investigación del valor de posición parece orientarse actualmente en una nueva dirección, que intenta develar cómo ocurre el procesamiento numérico de este principio en nume- rales multidígitos (ver Kallai \& Tzelgov, 2012; Kracjcsi \& Szabó, 2012). Así, Kallai y Tzelgov (2012) sugieren que los dígitos - del 1 al 9-en los numerales arábigos constituyen un conjunto de elementos básicos que se encuentran fuertemente asociados con sus significados, los cuales corresponden a representaciones de cantidades pequeñas. Así, esta cualidad de los dígitos les permitiría ser automáticamente procesados. En este sentido, los autores plantean que otras características estructurales de los numerales también son incluidas en este procesamiento, tal como lo es el valor de posición. Sin embargo, los resultados logrados hasta el momento no son concluyentes y la hipótesis mencionada deberá ser explorada en investigaciones futuras.

\section{Referencias}

Baturo, A. R. (2000). Construction of a numeration model: A theoretical analysis. En J. Bana \& A. Chapman (Eds.), Proceedings 23rd Annual Conference of the Mathematics Education Research Group of Australia (pp. 95-103). Fremantle, WA.

Becker, J. \& Várelas, M. (1993). Semiotic aspects of cognitive development: Illustrations from early mathematical cognition. Psychological Review, $100(1), 420-431$

Bedoya, E. \& Orozco, M. (1991). El niño y el sistema de numeración decimal. Comunicación, Lenguaje y Educación, 11(12), 55-62.

Bedoya, N. M. (2013). Comprensión del valor de posición y composición numérica. Memorias, 11(20), 99-110.

Bedoya, N. M., Muñoz, B., \& Medina, D. A. (2012). Evaluación, diagnóstico e intervención en la comprensión del valor de posición. En G. Obando (Ed.), $13^{\circ}$ Encuentro Colombiano de Educación Matemática (pp. 1038-1044). Medellín, $\mathrm{CO}$.

Boulton-Lewis, G. M. (1993). An analysis of relation between sequence counting and knowledge 
of place values in the early years of school. Mathematics Education Research Journal, 5(2), 94-106.

Broadbent, A. (2004). Understanding place-value. A case study of base ten game. Australian Mathematics Classroom, 8(4), 45-46.

Byrge, L., Smith, L. B., \& Mix, K. S. (2014). Beginnings of place value: How preschoolers write three-digit numbers. Child Development, 85(2), 137-443. doi: 10.1111/cdev.12162

Cadavid, G. S. (2013). Enseñanza del valor posicional en el sistema de numeración decimal para niños de escuela básica usando las nuevas tecnologías (Tesis de maestría, Universidad Nacional de Colombia, Medellín, Colombia). Recuperado de http://www.bdigital.unal.edu. co/9841/1/43985819.2013.pdf

Cawley, J. F., Parmar, R. S., Lucas-Fusco, L. M., Kilian, J. D., \& Foley, T. E. (2007). Place value and mathematics for students with mild disabilities: Data and suggested practices. Learning Disabilities: A Contemporary Journal, 5(1), 21-39.

Ceballos, F. A. (2012). Comprensión del valor de posición a partir de la relación del conteo "a partir de", la composición aditiva y la equivalencia numérica; un estudio exploratorio en niños de $2^{\circ}$ de primaria (Trabajo de grado en Psicología, Universidad del Valle, Cali, Colombia).

Chang, W. W-L., Au, T. K., \& Tang, J. (2014). Strategic counting: A novel assessment of place-value understanding. Learning and Instruction, 29(1), 78-94. doi: http://dx.doi.org/10.1016/j.learninstruc.2013.09.001

Chan, B. M. Y. \& Ho, C. S. H. (2010). The cognitive profile of Chinese children with mathematics difficulties. Journal of Experimental Child Psychology, 107(1), 260-279. doi: 10.1016/j. jecp.2010.04.016

Cotter, J. A. (2000). Learning place value in first grade through language and visualization. Teaching Children Mathematics, 7(2), 108-114.

Fuson, K. C. (1990a). Conceptual structures for multiunit number: implications for learning and tea- ching multidigit addition, subtraction, and place value. Cognition and instruction, 7(4), 343-403.

Fuson, K. C. (1990b). Issues in place-value and multidigit addition and subtraction learning and teaching. Journal for Research in Mathematics Education, 21(4), 273-280.

Fuson, K. C. (1998). Pedagogical, mathematical, and real-world conceptual-support nets: A model for building children's multidigit domain knowledge. Mathematical Cognition, 4(2), 147-186.

Fuson, K. C. \& Briars, D. J. (1990). Using a base-ten blocks learning/teaching approach for first- and second- grade place-value and multidigit addition and subtraction. Journal for Research in Mathematics Education, 21(3), 180-206.

Fuson, K.C., Wearne, D., Hiebert, J., Murray, H., Human, P., Olivier, A., Carpenter, T., \& Fennema, E. (1997). Children's conceptual structures for multidigit numbers and methods of multidigit addition and subtraction. Journal for Research in Mathematics Education, 28, 130-162.

Hederich, C. \& Camargo, A. (2002). Hacia la construcción de un modelo de procesamiento numérico. El desarrollo de la transcodificación de numerales verbales a formato arábigo (Informe Técnico Final Presentado a Colciencias). Bogotá: CIUP, Universidad Pedagógica Nacional.

Hiebert, J. \& Carpenter, T. P. (1992). Learning and teaching with understanding. En D. A. Grouws (Ed.), Handbook of research on mathematics teaching and learning (pp. 65-97). Nueva York: Macmillan.

Hiebert, J., \& Wearne, D. (1992). Links between teaching and learning place value with understanding in first grade. Journal for Research in Mathematics Education, 23(2), 98-122.

Hiebert, J. \& Wearne, D. (1996). Instruction, understanding, and skill in multidigit addition and subtraction. Cognition and Instruction, 14(3), 251-283.

Ho, S.-H. \& Cheng, F. S.-F. (1997). Training in place-value concepts improves children's addition 
skills. Contemporary Educational Psychology, 22(1), 495-506.

Hugues, M. (1986). Children and number. Difficulties in learning mathematics. Oxford: Blackwell Publishing Ltd.

Hunter, J. \& Turner, I. (1994). Learning multi-unit number concepts and understanding decimal place value. Educational Psychology, 14(3), 269-282. doi:10.1080/0144341940140302

Johansson, B. S. (2005). Numeral writing skill and elementary arithmetic mental calculations. Scandinavian Journal of Educational Research, 49(1), 3-25.

Jones, G. A., Thornton, C. A., \& Putt, I. J. (1994). A model for nurturing and assessing multidigit number sense among first grade children. Educational Studies in Mathematics, 27(1), 117-143.

Kallai, A. Y. \& Tzelgov, J. (2012). The place-value of a digit in multi-digit numbers is processed automatically. Journal of Experimental Psychology: Learning, Memory, and Cognition, 38(5), 1221-1 233. doi:10.1037/a0027635

Kamii, C. K. (1985). El niño reinventa la aritmética: Implicaciones de la teoría de Piaget. Barcelona: Aprendizaje-Visor.

Kracjcsi, A. \& Szabó, E. (2012). The role of number notation: sign-value notation number processing is easier than place-value. Frontiers in Psychology, 3(1), 1-15. doi: 10.3389/fpsyg.2012.00463

Lamon, S. (1999). Teaching fractions and ratios for understanding: Essential content knowledge and instructional strategies for teachers. Mahwah, NJ: Lawrence Erlbaum.

Lerner, D. \& Sadovsky, P. (1994). El sistema de numeración: un problema didáctico. En C. Parra y J. Saiz (Eds.), Didáctica de las matemáticas (pp. 95-84). Buenos Aires: Editorial Paidós Educador.

Medina, D. A. (2012). Efecto de la comprensión del valor de posición en la escritura de numerales de niños en $1^{\circ}$ grado (Tesis de maestría en Psicología, Universidad del Valle, Cali, Colombia).
Medina, D. A. (2013). Evaluación del cambio en la producción de numerales arábigos. En Y. Morales \& A. Ramírez (Eds.), Memorias del I Congreso de Educación Matemática de América Central y El Caribe (pp. 796-807). Santo Domingo, DO.

Meel, D. E. (2003). Modelos y teorías de la comprensión matemática: Comparación de los modelos de Pirie y Kieren sobre el crecimiento de la comprensión matemática y la Teoría APOE. Revista Latinoamericana de Investigación en Matemática Educativa, 6(3), 221-278.

Miura, I. T., Okamoto, Y., Kim, C. C., Steere, M., \& Fayol, M. (1993). First graders' cognitive representation of number and understanding of place value: Cross-national comparisons-France, Japan, Korea, Sweden, and the United States. Journal of Educational Psychology, 85(1), 24-30.

Nunes, T. \& Bryant, P. (1997). Las matemáticas y su aplicación: La perspectiva del niño. México D.F.: Siglo XXI Editores.

Orozco, M. (1994). El sistema notacional en base diez. Recuperado de http://cognitiva.univalle. edu.co/archivos/grupo $\% 20$ matematica $\% 20$ y\%20cognicion/Mariela/capitulos/cap5.pdf

Orozco, M., Guerrero, D. F., \& Otálora, Y. (2007). Los errores sintácticos al escribir numerales en rango superior. Infancia y Aprendizaje, 30(2), 147-162.

Price, P. (1999). Using place-value blocks or a computer to teach place-value concepts. En I. Schwank (Ed.), Proceedings of the First Conference of the European Research in Mathematics Education (pp. 259-269). Osnabrück, DE.

Price, P. S. (2001). The development of year 3 students' place value understanding: Representations and concepts (Tesis doctoral, Queensland University of Technology, Brisbane, Australia). Recuperado de http://eprints.qut.edu. au/15783/1/Peter_Price_Thesis.pdf

Ross, S. H. (abril, 1986). The development of children's place-value numeration in grades two 
through five. Annual Meeting of the American Educational Research Association, San Francisco, CA. Recuperado de http://files.eric.ed.gov/ fulltext/ED273482.pdf

Ross, S. H. (1990). Children's acquisition of place-value numeration concepts: The roles of cognitive development and instruction. Focus on Learning Problems in Mathematics, 12(1), 1-17.

Ross, S. H. (2002). Place value: Problem solving and written assessment. Teaching Children Mathematics, 8(7), 419-423.

Saxton, M. \& Cakir, K. (2006). Counting-on, trading and partitioning: Effects of training and prior knowledge on performance on base- 10 tasks. Child Development, 77(3), 767-785. doi: 10.1111/j.1467-8624.2006.00902.x

Sinclair, A. \& Scheuer, N. (1993). Understanding the written number system: 6 year-olds in Argentina and Switzerland. Educational Studies in Mathematics, 24(2), 199-221.

Thompson, I. (2000). Teaching place value in UK: time for a reappraisal? Educational Review, 52(3), 291-298.

Várelas, M. \& Becker, J. (1997). Children's developing understanding of place value: Semiotic aspects. Cognition and Instruction, 15(2), 256-286.
Fecha de recepción: agosto 26, 2014

Fecha de aceptación: febrero 1, 2016 